\title{
Spouse Caregivers' Attitudes Toward Obtaining a Diagnosis of a Dementing Illness
}

\author{
Cathleen M Connell, PhD, and Mary P. Gallant, MPH, PhD ${ }^{\dagger}$
}

OBJECTIVES: To examinc attitudes of spouse caregivers about the process of obtaining a diagnosis of a dementing illness, including perceived benefits and obstacles to obtaining a diagnosis and suggestions for improving the process.

DESIGN: A mail survey of spouse caregivers of dementia patients followed by the Michigan Alzheimer's Disease Research Center.

PARTICIPANTS: The 233 spouse caregivers of dementia patients who returned a completed questionnaire.

RESULTS: More than three-fourths of the sample rated the following benefits of obtaining a diagnosis as very or extremely important: ruling out other causes of memory or behavior problems, allowing family members to get information about dementia, may qualify my spouse for drug treatment, and will allow me to plan for the future. Primary obstacles included the time required to obtain a diagnosis, the cost of procedures, lack of access to physicians trained to diagnose dementing illnesses, and not receiving a referral from a primary care physician $(41.6 \%, 41.0 \%, 40.8 \%$, and $24.1 \%$ of the sample, respectively, agreed or strongly agreed that the factor posed a barrier to obtaining a diagnosis). Content analysis of responses to two open-ended questions identified problematic or stressful aspects of obtaining a diagnosis and suggestions for making the process easier for the patient and family.

CONCLUSION: Results suggest the need to provide an orientation to the diagnosis and assessment process for patients and family members, develop a training session for physicians to better prepare them to address the emotional needs of patients and families and to disclose the diagnosis in an informative and compassionate manner, and provide outreach education to primary care physicians to increase knowledge and awareness of dementing illnesses and to increase referrals to community-based services and health professionals able to address the concerns of family members. J Am Geriatr Soc 44:1003-1009, 1996.

From the "School of Public Health and the Michigan Alzheimer's Disease Re search Center, University of Michigan, and the tSchool of Social Work, University of Michigan, Ann Arbor, Michigan

Funding for this research was provided by the U.S. Public Health Service, NIA P.50-AG08671, to the Michigan Alzheimer's Discase Research Center.

Address correspondence to Cathleen M Connell, PhD, Michigan Alzheimer's

Disease Research Center, 300 North Ingalls Bldg., Rm 3D03, University of

Michigan, Ann Arbor, University of Michigan, Ann Arbor, MI 48109-0489.
A great deal of research has been conducted to examine Athe stress of providing full-time care to a family member with a dementing illness. ${ }^{\mathrm{I}-3}$ Less attention has been paid to attitudes about obtaining a diagnosis of a dementing illness, despite the potential role a diagnosis may play in family adaptation and coping. For some families, a diagnosis provides a sense of relief because disturbing behaviors can be attributed to a disease process that is not under the affected person's voluntary control. ${ }^{4} \mathrm{~A}$ diagnosis may facilitate access to supportive services (i.e., adult day care, respite care), promote identification with families who are facing similar problems, ${ }^{5}$ and enhance communication among patients, family members, and health professionals. ${ }^{6}$ A specific and precise diagnosis and resulting information about prognosis may help families manage behavioral problems, adapt to progressive changes as they occur, and facilitate the implementation of advance directives and long-term planning. ${ }^{5}$ Most families that obtain a diagnosis feel that it is beneficial ${ }^{5}$ and would choose to be informed of the diagnosis if their physicians suspected a dementing illness. ${ }^{7}$ In a study of predictive testing for Huntington's disease, knowing what to expect in the future has been shown to have potential benefits for psychological health by reducing uncertainty and enhancing quality of life. ${ }^{8}$

For other families, however, obtaining a diagnosis may interfere with optimal adaptation and coping by confirming a chronic, progressive illness associated with mental and physical decline. ${ }^{4}$ As a result of obtaining a diagnosis, family members may need to confront difficult emotional issues related to the disease because they are no longer able to deny symptoms. ${ }^{4}$ Existing family relationships and roles may need to change as the patient assumes an increasingly dependent role. ${ }^{9}$ In some cases, the roles of caregiver and care recipient may be reversed (i.e., an adult child assuming increasing responsibility for the care of a parent) or the caregiver may become responsible for unfamiliar tasks (e.g., cooking, financial management).

Some family members fear that a diagnosis will destroy their ability to live a normal life and prefer to hope for the best rather than know the worst. ${ }^{10}$ As reported by a wife about her husband's diagnosis of a dementing illness, "I cope with my feelings about the disease as I do with any other unpleasant intrusion into my life - by ignoring them". ${ }^{10}$ Even when physicians explain the diagnosis and prognosis in a sensitive way, some families are unable or unwilling to assimilate this information, ${ }^{11}$ especially because the diagnosis cannot be made with absolute certainty. ${ }^{12}$ Instead, patients and family members erect walls of defense that prevent them from 
coming to terms with the possible implications of a dementing illness. ${ }^{13}$ Haley et al. ${ }^{14}$ report that a sample of family caregivers waited an average of 3 years from onset of symptoms to seek a medical evaluation related to memory loss. Aside from the potential positive and negative impacts of a diagnosis of a dementing illness, the process of obtaining such a diagnosis may have unintended consequences for both patients and families. Because the assessment and diagnosis process is time consuming, can involve multiple tests and visits to specialists, and may lack a definitive outcome, patients and family members may experience a wide variety of emotional, physical, and financial stressors.

In addition to the impact of a diagnosis on the patient and family, the physician also experiences the burden of a diagnosis of a chronic debilitating illness. ${ }^{12}$ To avoid the family's sense of hopelessness and grief associated with the long-term implications of the disease, physicians may limit the amount of information they provide about a dementing illness ${ }^{5}$ or avoid making a specific diagnosis because they have difficulty delivering bad news, especially in light of the limited treatment options and generally poor prognosis. ${ }^{15}$ Families frequently report that physicians provide very limited information about the disease once diagnosed, few referrals to community-based services, ${ }^{14,16}$ and inadequate assistance with difficult family decisions related to the progression of the disease (e.g., management of difficult behaviors and long-term care needs). ${ }^{5,14,17}$

The overall goal of this exploratory study was to examine attitudes about diagnosis and about the process of obtaining a diagnosis of a dementing illness from the perspective of spouse caregivers. The specific aims of the study were to: (1) assess perceived benefits of obtaining a diagnosis for a spouse exhibiting symptoms of a dementing illness, (2) assess perceived obstacles to obtaining a diagnosis, (3) identify aspects of the process of seeking a diagnosis that are especially stressful, and (4) identify ways to make the process of obtaining a diagnosis easier for patients and spouses. Ultimately, results of the study may inform strategies for disclosing a diagnosis of a dementing illness and the development of educational interventions designed to facilitate family adaptation and coping.

\section{METHODS}

Data analyzed for this study were obtained from a mail survey of spouse caregivers of patients with a variety of dementing illnesses followed by the Michigan Alzheimer's Disease Research Center (MADRC). The MADRC, located at the the University of Michigan Medical Center, provides primary, secondary, and tertiary care mainly to the Ann Arbor community and residents of the state of Michigan.

Potential study participants were identified from the MADRC clinical database and included all co-dwelling spouses of noninstitutionalized patients with a diagnosis of dementia (excluding those who specifically expressed a disinterest in participating in research). The mailed survey was administered according to the Dillman Total Design Method. ${ }^{18}$ This method, which maximizes response rate, prescribes an initial survey mailing, a reminder postcard sent to all potential respondents 1 week later, a second survey mailing to all nonrespondents 3 weeks after the initial mailing, and a final survey mailing to the remaining nonrespondents 7 weeks after the initial mailing. Return postage was provided for all mailings. Of 355 individuals who were identified as meeting the criteria for inclusion, 233 returned a completed questionnaire, representing a response rate of $66 \%$.

The final sample of 233 spouse caregivers consisted of 109 men (47\%) and 124 women (53\%). The sample ranged in age from 30 to 92 , with a mean age of 68 . The majority $(91 \%)$ of respondents were white. Approximately $90 \%$ of the sample had at least a high school diploma, and one-third had a college degree. Eighty-six percent of the respondents had been married more than 30 years, and two-thirds had been married more than 40 years. The majority $(56.1 \%)$ had provided care for their spouse for 4 years or less; $44 \%$ had provided care for 5 years or more. More than one-half of the sample was providing care to a spouse with Alzheimer's disease $(54 \%)$; others cared for spouses with unspecified cognitive disorder $(21 \%)$, Huntington's disease $(8 \%)$, Parkinson's disease with dementia $(7 \%)$, mixed cognitive disorder $(5 \%)$, multi-infarct dementia $(3 \%)$, and progressive supranuclear palsy $(2 \%)$.

Several questions about attitudes toward diagnosis were included in the survey. The first item included in the mailed survey asked respondents to rate the importance of eight potential benefits of obtaining a diagnosis that have been identified in previous literature. ${ }^{4,5,12}$ The benefits were listed under a stem "Getting a diagnosis of dementia ...." and included: (1) will let me know what is wrong with my spouse, (2) will allow me to plan for the future, (3) will allow me to involve my spouse in important decisions (e.g., making a will), (4) will allow me to get information about dementia, (5) may qualify my spouse for drug treatment, $(6)$ will be helpful to my family in case dementia is hereditary, (7) will allow me to rule out other causes of memory or behavior problems, and (8) will allow me to use appropriate community services. Five response choices were offered, including "not at all important," "a little important," "somewhat important," "very important," and "extremely important."

Items that assessed perceived obstacles to obtaining a diagnosis were based on results of 11 focus group interviews conducted with family caregivers as part of a communitybased educational outreach intervention. ${ }^{19,20}$ Two questions designed for the present study were based on themes identified from the analysis of the interviews and assessed obstacles to obtaining a diagnosis. Respondents indicated the extent to which they agreed with seven statements that were listed under a stem "Getting a diagnosis of dementia for my spouse was difficult because. . ." (1) my family physician did not make a referral to a specialist, (2) there are so few physicians trained to diagnose dementia in my local area, (3) the process is so time consuming, (4) the process is not covered by our health insurance, (5) my family members didn't agree that it was important, and (6) my spouse was not willing to be tested. Response choices included "strongly disagree," "disagree," "neutral," "agree," and "strongly agree."

Respondents were also asked to indicate the extent to which they agreed with nine statements that were listed under a stem "Getting a diagnosis of dementia is more trouble than it is worth because. .. "(1) it is very demanding for the patient, (2) it is very demanding for the family, (3) so little can be done for people with dementia, (4) memory problems are part of the normal aging process, (5) there is no cure for dementia, (6) there is no effective treatment, (7) there is a stigma against people with dementia, (8) it is easier not to know what the diagnosis is, and (9) it would require seeing a 
specialist instead of a family physician. Response choices included "strongly disagree," "disagree," "neutral," "agree," and "strongly agree." Freqencies, response rates, and chi-square analyses used to examine patterns of nonresponse were generated using the Statistical Package for the Social Sciences.

Two open-ended questions were also included in the survey: "What steps to getting a diagnosis were problematic or stressful for you?" and "Do you have any suggestions for how to make the process of getting a diagnosis easier for the patient and family?" Respondent's written comments, recorded in space provided in the survey instrument, were transcribed verbatim. These comments reflected caregiver's experiences about the MADRC as well as diagnostic and assessment services provided in local communities throughout Michigan. Content analysis, defined as "a research technique for making replicable and valid inferences from data to their context" ${ }^{21}$ was used to analyze the transcripts. Predominant themes were selected as the unit of analysis and quotes were selected to illustrate each theme, according to accepted procedures for this qualitative data analysis technique. ${ }^{21,22}$

\section{RESULTS}

Benefits of and Obstacles to Obtaining a Diagnosis

Participants' ratings of the importance of eight possible benefits of obtaining a diagnosis are included in Table $1 \mathrm{~A}$. Participants were asked to indicate the extent to which they agreed that seven factors served as obstacles to obtaining a diagnosis and to indicate the extent to which they agreed that nine factors outweighed the benefits of obtaining a diagnosis. Results are presented in Tables $1 \mathrm{~B}$ and $1 \mathrm{C}$, respectively. Missing data for these ratings ranges from $5.6 \%$ to $11.2 \%$ (13 to 26 cases) for the 233 study participants. Patterns of nonresponse were not significantly related to demographic or health background characteristics of the sample. Percentages of respondents rating each item as "very important" or "extremely important" (for Table 1A) and "agree" or "strongly agree" (for Tables 1B and 1C) are combined to facilitate the interpretation of the relative rankings of the benefits of and obstacles to obtaining a diagnosis.

\section{Problematic or Stressful Aspects of Obtaining a Diagnosis}

Almost three-fourths of the sample $(70 \%)$ answered the first open-ended question: "What steps to getting a diagnosis

Table 1. Ratings of the Importance of Selected Benefits of Obtaining a Diagnosis, Respondents Agreement that Selected Obstacles Posed Difficulties to Obtaining a Diagnosis, and Respondents Agreement that Selected Factors Outweighed the Benefits of Obtaining a Diagnosis $(\mathbf{N}=233)$

A) Benefits Very/Extremely Important

Getting a diagnosis of dementia ...

- will let me know what is wrong with my spouse $(n=220)$

- will allow me to rule out other causes of memory or behavior problems $(n=216)$

- will allow me to get information about dementia $(n=216)$

$87.7 \%$

$81.5 \%$

- may qualify my spouse for drug treatment $(n=217)$

$80.1 \%$

$77.4 \%$

- will allow me to plan for the future $(n=220)$

- will be helpful to my family in case dementia is hereditary $(n=219)$

$76.3 \%$

- will allow me to involve my spouse in important decisions $(n=215$ )

$73.5 \%$

- will allow me to use appropriate community services $(n=216)$

$68.8 \%$

$66.7 \%$

B) Obstacles

Agree/Strongly Agree

Getting a diagnosis of dementia for my spouse was difficult because ...

- the process is so time consuming $(n=209)$

$41.6 \%$

- the process is so expensive ( $n=207$ )

- there are so few physicians trained to diagnose dementia in my local area $(n=213)$

$41.0 \%$

- my family physician did not make a referral to a specialist $(n=212)$

- my spouse was not willing to be tested $(n=212)$

- the process is not covered by our health insurance $(n=207)$

$40.8 \%$

$24.1 \%$

$15.6 \%$

$12.6 \%$

- my family members didn't agree that it was important $(n=210)$

$6.2 \%$

\section{C) Factors}

Agree/Strongly Agree

Getting a diagnosis of dementia is more trouble than it is worth because ...

- there is no cure for dementia ( $n=212$ )

$49.0 \%$

- it would require seeing a specialist instead of a family physician ( $n=214$ )

$45.4 \%$

- there is no effective treatment $(n=207)$

$44.5 \%$

- so little can be done for people with dementia ( $n=211$ )

$37.9 \%$

- there is a stigma against people with dementia $(n=208)$

$34.7 \%$

- it is very demanding for the patient $(n=213)$

$23.5 \%$

- memory problems are part of the normal aging process $(n=215)$

$22.3 \%$

- it is very demanding for the family $(n=213)$

$21.6 \%$

- it is easier not to know what the diagnosis is $(n=211)$ 
were problematic or stressful for you?" Compared with the sample as a whole, respondents who answered this question were more likely to be female than male $(P<.0001)$. Content analysis of all of the responses to this question identified five major themes: (1) finding a physician with expertise in dementing illnesses, (2) diagnostic and assessment procedures, (3) the manner in which the diagnosis was disclosed to the patient and family, (4) the perceived consequences of obtaining a diagnosis, and (5) benefits of obtaining a diagnosis.

\section{Finding a Physician}

Several respondents mentioned having problems finding a physician qualified to provide a diagnosis, in part, because their family physician was not trained to do so and did not provide an appropriate referral.

"We were informed by our family doctor that there is nothing that can be done. We didn't know where to go from there."

"Our family doctor only said 'you are getting older so you must expect these things.' He (the patient) was 71 years old then. We then saw a neurologist."

"The worst part was the feeling of having to fight with the HMO for two to three years before being able to see someone knowledgeable in this area."

\section{Diagnostic and Assessment Procedures}

For many caregivers, the emotional and financial cost of testing seemed to ourweigh the possible benefits of obtaining a diagnosis.

"Our family physician diagnosed Alzheimer's almost on the first visit. We then went (to specialists) for more tests. It got so expensive and was not really belping the condition, so we just quit going for more tests."

"When I understood his memory loss, I found no logical reason to pursue more tests. An eight-bour test would not tell me any more than I knew about his memory loss."

Other respondents mentioned the long wait to get an initial appointment, the long wait between doctor's appointments, the long delay in obtaining a diagnosis once testing was complete, and the time spent waiting in the hospital between tests.

Some respondents reported that having a physician repeat diagnostic tests that had been completed previously was very stressful:

"The same tests were performed at two different hospitals. One hospital wouldn't accept the other hospital's tests, therefore, they had to be repeated. Having to return so many times was stressful. The first hospital gave a definite diagnosis and left it at that - they didn't tell us where else to turn. I had to suggest a second opinion."

Several caregivers mentioned that the process of obtaining a diagnosis was upsetting because family members could not be with the patient during testing. Others reported being anxious because they didn't know how much to discuss with the patient and that they felt more hopeless with every test result. One caregiver mentioned that the testing process in general was stressful because it exposed the patient's limitations and cognitive deficits:

"He cried when be could not do the simple exercises and thought if he read and studied more his memory would improve."

\section{Manner In Which the Diagnosis Was Disclosed}

For some caregivers, the manner in which the diagnosis was disclosed to the patient and family was the most difficult part of the process. Several quotes are particularly illustrative:
"We were given a clinical, sterile, unfeeling and detached diagnosis, lacking human compassion. It left us very devastated, there was little understanding or caring of our emotional and psychological reactions."

"We experienced difficulty in getting a proper sympathetic diagnosis for my husband. It was handled very callously and was very detrimen. tal to my husband's health."

"The specialist told us that my husband has Alzheimer's disease and to leave the room so that the doctor could talk to me (the patient's wife). Then he (the physician) told me to look in the phone book and call neurologists to see if any of them were planning any drug trials. He (the physician) couldn't be bothered to find a referral for us. I found this very inhumane."

"Too much dragging out and stress and uorry and waiting for the family. If you're not sure of the diagnosis, say so, then the family can plan and decide what to do. If nothing can be done, say so!"

"Hearing 'we think it sounds like Alzheimer's' is the last thing the family wants to hear. Please show a little compassion and consideration for the patient and gently present the final diagnosis after the family is prepared to bear the word 'Alzheimer's' and is ready to accept it."

One caregiver mentioned the lack of opportunity to discuss the diagnosis privately with the physician.

"It was hard to have my husband sitting in the same room and have the doctors discussing his illness in front of him."

\section{Perceived Consequences of the Diagnosis}

After obtaining a diagnosis, several respondents expressed their disappointment that their physician didn't provide referrals to community-based agencies that could provide assistance. One caregiver reported that the diagnosis increased her depression and anxiety. Several respondents mentioned that they expected to receive a written report to confirm the diagnosis after testing was completed but were disappointed when the report never arrived. Other respondents mentioned the stress of needing to accept that something is wrong and that little can be done.

"I often wish there had been no diagnosis because there wasn't any medications to help and insurance doesn't kick in. If the diagnosis is stroke or a vascular problem, at least patients aren't treated as if they are psychotic."

"We had no difficulty getting a diagnosis, just grave disappointment when we learned there was no relief of symptoms."

\section{Benefits of Obtaining a Diagnosis}

Several benefits of obtaining a diagnosis emerged from the content analysis. For some respondents, the diagnosis provided a sense of relief because it helped the family to know what to expect and confirmed that they were doing all that they could.

"The trips (to the specialist) were tiring and time-consuming, but we were glad we went. It was helpful to know what to expect and that I wasn't neglecting him if there was anything that could be done for bim."

"The steps were not problematic, but facing the unknown during the testing was stressful. The diagnosis was heartbreaking at first, but having a name for the problem and then proper treatment gave a feeling of relief."

"I don't regret going for all of the tests to find out just what was wrong. It was very stressful, but so is the whole disease. The worst thing is the pain my husband had trying to do things during the testing and failing, but it is all necessary." 
Suggestions for Making the Process of Obtaining a Diagnosis Easier for the Patient and Family

Less than one-half of the sample (44\%) answered the open-ended question: "Do you have any suggestions for how to make the process of getting a diagnosis easier for the patient and family?" There were no demographic or health background differences between respondents and nonrespondents to this question. Three major themes were identified as a result of a content analysis of the responses to this question: (1) involving family physicians in the diagnostic and assessment process, (2) streamlining diagnostic and assessment procedures, and (3) involving family members in the diagnosis and assessment process.

\section{Involving Family Physicians in the Diagnosis and Assessment Process}

Several respondents mentioned the importance of increasing knowledge and awareness of dementing illnesses among family physicians.

"We need more doctors who are up-to-date on the disease and willing to be belpful after the patient has been diagnosed."

"Regular doctors should have more information on Alzheimer's and where and what to do to get help for the family. This is a family disease."

Caregivers also mentioned the importance of obtaining a referral to a specialist if their family physician was not trained to conduct a comprehensive diagnosis and assessment.

"Some doctors diagnose people as having senile dementia without giving full testing. It would be helpful if doctors who suspect senile dementia would please refer the patient to a center where they can receive proper, full testing and any treatment possible."

Several respondents felt that physicians should encourage families to pursue a comprehensive diagnosis and assessment and emphasize the benefits of knowing what is wrong.

"We should know that having a complete work-up at a medical center early on saves time, money, and energy. Learning early how to cope with memory loss simplifies life. Accepting the situation, dealing with the difficult things (no more driving, handling money, and finances) can reduce the stress levels of both caretaker and patient."

"If a person could know when a loved one has Alzheimer's early enough, they could be put on some medicine. My wife was too far mentally advanced for medication when she was seen (at a memory disorders clinic)."

\section{Streamlining Diagnostic and Assessment Procedures}

Respondents mentioned several strategies to streamline the diagnostic and assessment process, thereby making it easier for patients and families: avoid duplicating tests, develop more accurate and easy to administer diagnostic tests, have the same specialist administer all diagnostic tests, increase flexibility in setting up appointments, provide transportation, reduce the number of tests, and offer testing in the family physician's office. Several respondents suggested that the testing process be less time consuming, perhaps being completed in one visit.

"When you have to drive a distance, as we did, it would be nice for everything to be scheduled for one day, with a report the following week, instead of spread out as our appointments were, making it more stressful for everyone."

"We had to stay overnight a few times because he had to be seen by different people and that was difficult for him, but we managed to stay. He gets lost in strange places, so be wanted to go out in the hall all the time to find his "friends."
One caregiver suggested that more research is needed to simplify and standardize testing procedures.

"We clearly need research to develop a medically accurate lab test (blood or tissue). This may take several years, but it could also lead to finding a root cause and ultimately a cure or at least some way to prevent the onset of the disease. Meanwhile there should be a standard testing protocol established for all who have the symptoms and approved facilities to provide the tests."

\section{Involving the Family in the Diagnosis and Assessment Process}

Many respondents mentioned the need to involve family members in all phases of the diagnostic and assessment process.

"After the first interview, my husband would not permit me to participate in interviews with the doctor. Therefore, she (the physician) had only the patient's view of what was going on. He (the patient) can be very clever and deceptive. I know some of the things he told her, which, without the supporting physical evidence of the tests, convinced her that he was perhaps only depressed. She finally closed bis file without making a diagnosis. I feel that the caregiver should be given equal time with the physician. A person who is disoriented, confused, and forgetful is hardly the one to trust. In our case, my husband is always right, very defensive, and extremely smart."

"Have the spouse be with the patient when he/she sees the doctors."

"Listen to the patient and the family when they indicate something is wrong or a treatment is not working."

Respondents suggested that all test results be communicated to the family and that physicians should respect the family's preference for how information is communicated.

"Not having the doctors talk about the problem in front of the patient, especially in the beginning."

"Provide a summary for family and patient to read concerning the process as to how diagnosis is made."

"Talk to both spouses together, treat the patient with dignity and try and reassure him/her that be (the physician) will belp in any way that be can - or recommend a specialist in this field who can help."

One respondent recommended that physicians employ a patient coordinator to help families understand the results of the diagnostic and assessment process.

"There should be a patient coordinator who counsels and helps put it all together - someone who discusses results and its meaning with the non-dementia spouse. Then a tender loving treatment of the dementia victim, revealing only what's needed to cope with the particular phase of the disease."

\section{DISCUSSION}

The majority of respondents endorsed the benefits of obtaining a diagnosis, including finding out what is wrong and ruling out other causes of memory or behavior problems. A diagnosis also helped families to plan for the future because it triggered discussion of treatment options, the use of community-based services, and legal and financial matters. For many caregivers, a diagnosis provided a sense of relief because it helped the family to know what to expect and confirmed that they were doing all that they could for their spouse.

Some respondents also experienced obstacles to obtaining a diagnosis, in particular the time consuming and expensive nature of the process. Other barriers to obtaining a diagnosis included the beliefs that there is no cure or treatment for dementia and that memory problems are part of normal aging, and the preference for receiving care from a family physician rather than a specialist. Both structural and 
emotional aspects of the process of obtaining a diagnosis were perceived by the spouse caregivers as problematic. For example, caregivers reported difficulties finding a physician in their community qualified to conduct a comprehensive assessment. After receiving a referral to a physician, respondents reported problems in obtaining an initial appointment and then scheduling subsequent visits to complete testing. Once a diagnosis was given, many caregivers reported that they didn't know where to turn or what to do next, resulting in increased anxiety, stress, and depression. For some caregivers, the manner is which the diagnosis was given was perceived as lacking in sympathy and compassion.

As a result of participants' comments to the open-ended questions included in the survey, several suggestions for improving the process and overcoming the obstacles to obtaining a diagnosis can be offered. An orientation to the diagnosis and assessment process would be very beneficial for the patient and family and could be provided by a nurse, health educator, or social worker in collaboration with a geriatric assessment team. Such an orientation could include: (1) a rationale for and description of each test performed and how each contributes to an understanding of the disorder or disease, (2) a projected timeline depicting when all tests will be completed and when results will be shared with the family, (3) an assessment of the accuracy of a diagnosis, (4) a rationale for including or excluding family members from testing procedures, and (5) a set of written materials that describe all phases of the diagnostic and assessment process and are appropriate for varied literacy levels.

Because of the varied personal meanings of disease and diagnosis, results of a comprehensive psychosocial evaluation of the patient and family could be very informative to the physician who ultimately discloses the diagnosis. As part of this evaluation, the patient and family could articulate their preferences for when and the way in which the diagnosis is disclosed. For example, some caregivers may prefer to hear the diagnosis before the patient so that they can control their emotions and be more supportive when the patient is first informed. A recent survey indicates that primary care physicians may be willing to accommodate this preference; $90 \%$ of a sample of 498 primary care physicians reported that they are very likely to inform an adult daughter of a parent's diagnosis of a dementing illness, whereas only one-half of the physicians reported that they are very likely to disclose the diagnosis to the parent directly. ${ }^{2.3}$ Knowing what to expect and having some input into the diagnostic and assessment process may greatly reduce the stress for the patient, family member, and physician. In some cases, it may be best to delay the disclosure of a diagnosis until the patient and family have received counseling and are more prepared to accept the results of the assessment process. (For additional discussion of this issue, see references 12, 24, and 25).

Although all physicians are at times compelled to deliver bad news, few have had formal training to prepare them to respond adequately to the potentially profound impact of the experience. ${ }^{26}$ According to a random survey of 500 physicians, family issues related to the management of dementia were rated as among the most difficult clinical problems encountered. ${ }^{27}$ An educational program could be designed for physicians to increase their understanding of the emotional and psychological needs of patients and families during the diagnosis and assessment process and teach specific techniques to disclose the diagnosis in an informative and com- passionate manner. Such a program could be incorporated into medical school and continuing education curricula.

Ideally, the manner in which a diagnosis is disclosed would be tailored to the individual needs and coping style and capacity of the patient and family members. ${ }^{15,28}$ Responses to bad news vary considerably, depending on the meaning of the diagnosis and the patient's and family's previous experience with illness. ${ }^{2.5}$ The appropriateness of frank disclosure of medical information is also culture specific and may vary by race and ethnicity. For example, only one-third of physicians in Japan would reveal a diagnosis of cancer to their patients and only one-half of patients would choose to have the diagnosis revealed. ${ }^{29}$ Similarly, only $49 \%$ of cancer patients in Greece report that they want to be informed of their diagnosis, ${ }^{30}$ compared with $72 \%$ in a comparable study conducted in the United States. ${ }^{24}$

Primary care physicians can play an important role in countering myths and misconceptions about dementing illnesses (e.g., memory loss is a normal part of aging) and providing comprehensive diagnosis and assessment. ${ }^{23}$ Outreach education that targets physicians is needed to expand the network of service providers that respond appropriately to the needs of people with dementing illnesses and their family members. Outreach efforts are especially warranted because of the considerable range of primary care physicians' knowledge and understanding of dementia. ${ }^{23,31,32}$ Although most physicians take a patient history and provide or refer patients for physical, neurological, psychiatric, and neuropsychological examinations, few physicians use formal, published diagnostic criteria for dementing illnesses or use standardized mental status and cognitive function tests. ${ }^{2,3,3}$ Family physicians also need to be aware of and make referrals to community-based services (e.g., support groups, respite care programs, local chapter of the Alzheimer's Association) and to other professionals (e.g., social worker, case manager) who are able to discuss family members' concerns.

Several limitations to this study should be noted. Because there are no standardized measures of attitudes toward a diagnosis of a dementing illness, items were generated specifically for this study. Although the items performed well (i.e., moderate to high variability, no consistent patterns of missing responses) and were based on themes generated from extensive focus group interviews with family caregivers, some perceived benefits of and obstacles to obtaining a diagnosis may have been omitted.

The present sample was recruited from a patient database maintained by the Michigan Alzheimer's Disease Research Center. Because all respondents were providing care to a patient who had been referred to a specialist for diagnosis and assessment, the present sample is not representative of all families facing a dementing illness. In particular, the sample does not include those families who have chosen not to seek medical attention. For these families, the obstacles encountered may have outweighed any perceived benefits. On the other hand, the present sample is ideally suited for this exploratory study of attitudes toward a diagnosis among caregivers because each respondent directly experienced the process. Additional research is needed to examine issues related to the diagnosis and assessment of dementing illnesses with a larger and more racially and ethnically diverse sample of caregivers.

Learning that a spouse or parent has a progressive dementing illness for which there are limited treatment options 
and no cure is no doubt perceived as an extremely stressful experience. Indeed, dementing illnesses have been described as one of the most feared health problems of older adulthood. ${ }^{34}$ Additional research is needed to identify strategies to improve the diagnostic process to assure that patients and families are informed and knowledgeable about the assessment procedures and the disease, receive a diagnosis in a compassionate manner, and are referred to appropriate community-based services, including counseling, to facilitate optimal coping and adaptation.

\section{ACKNOWLEDGMENTS}

The authors thank the study participants and Norman Foster, Sara Kole, Sara Holmes, Peggy McGhan, Sid Gilman, and Christopher Benedict for their helpful comments on an earlier draft of this paper.

\section{REFERENCES}

1. Biegel DE, Sales E, Schulz R. Caregiving in Alzheimer's disease. In: Biegel DE, Sales E, Schulz R, eds. Family Caregiving in Chronic Illness, Newbury Park, CA: Sage Publications, 1991, pp 147-163.

2. Haley WE, Levine EG, Brown SL. Psychological, social, and health conscquences of caring for a relative with senile dementia. J Am Geriatr Soc 1987;35:405-411.

3. Schulz R, Visintainer P, Williamson GM. Psychiatric and physical morbidity effects of caregiving. J Gerontol 1990;45:P181-191.

4. Bass DM, McClendon J, Deimling GT, Mukherjee S. The influence of a diagnosed mental impairment on family caregiver strain. J Gerontol 1994;49:S146-155.

5. Glosser G, Wexler D, Balmelli M. Physician's and families' perspectives on the medical management of dementia. J Am Geriatr Soc 1985;33:383-391.

6. Centeno-Cortes C, Nunez-Olarte JM. Questioning diagnosis disclosure in terminal cancer patients: A prospective study evaluating patient's responses. Palliative Med 1994;4:39-44.

7. Erde EL, Nadal EC, Scholl TO. On truth telling and the diagnosis of Alzheimer's discase. J Family Pract 1988;26:401-406.

8. Wiggins $S$, Whyte $P$, Huggins $M$ et al. for the Canadian Collaborative Study of Predictive Testing. The psychological consequences of predictive testing for Huntington's disease. N Engl J Med 1992;20:1401-1405.

9. Chenoweth B, Spencer B. The experience of family caregivers. Gerontologist 1986;26:267-272.

10. Lynch HT, Harlan WL, Dyhrberg JS. Subjective perspective of a family with Huntington's Chorea. Arch Gen Psychiatry 1972;27:67-72.

11. Rabins PV. Management of dementia in the family context. Psychosomatics 1984;25:369.

12. Drickamer MA, Lachs MS. Should patients with Alzheimer's discase be told their diagnosis? N Engl J Med 1992;326:947-951.

13. Bahro M, Silber E, Sunderland T. How do patients with Alzheimer's disease cope with their illness?: A clinical experience report. J Am Geriatr Soc $1995 ; 43: 41-46$.
14. Haley WE, Clair JM, Saulsberry K. Family caregiver satisfaction with medical care of their demented relatives. Gerontologist 1993;32:219-226.

15. Wilson DB. A clinician's perspective on diagnostic research in Alzheimer's disease research: Ethical and legal issues. In: Berg JM, Karlinsky $H$, Lowy, FH, eds. Alzheimer's Discase Research: Ethical and Legal Issues. Ontario, Canada: Thomson Professional Publishing Canada, 1991, pp 263-272.

16. Fortinsky RH, Hathaway TJ. Information and service needs among active and former family caregivers of persons with Alzheimer's disease. Gerontologist 1990;30:604-609.

17. Martindale B Huntington's Chorea. Some psychodynamics seen in those at risk and in the responses of the helping professions. $\mathrm{Br} \mathrm{J}$ Psychiatry 1987;150:319-323.

18. Dillman DA. Mail and telephone surveys. New York: John Wiley \& Sons, 1978.

19. Connell CM, Kole SL, Benedict CJ et al. Increasing coordination of the dementia service delivery network: Planning for the Community Outreach Education Program. Gerontologist 1994;34:700-706.

20. Connell CM, Kole SL, Avey $\mathrm{H}$ et al. Attitudes about the dementia service delivery network: Attitudes about Alzheimer's disease and the dementia service delivery network among family caregivers and service providers in rural Michigan. Am J Alzheimers Disease 1996;11:15-25.

21. Krippendorff K. Content analysis: An introduction to its methodology. Beverly Hills, CA: Sage Publications, Inc., 1980.

22. Strauss A, Corbin J. Basics of qualitative research. Newbury Park, CA: Sage Publications, lnc, 1990

23. Fortinsky RH, Leighton A, Wasson JH. Primary care physician's diagnostic, management and referral practices for older persons and families affected by dementia. Res Aging 1995;17:124-148.

24. Estape JG, Palombo $H$, Hernandex $F$ et al. Looking for a better way to inform cancer patients. Proceedings of the Annual Meeting of the American Society of Clinical Oncology 1991;10:A1237.

25. Senra A, Palmeiro R, Garcia-Blanco P. Should the diagnosis of cancer be communicated to patients? Bull Cancer 1988;75:517-522.

26. Quill TE, Townsend P. Bad news: Delivery, dialogue, and dilemmas. Arch Intern Med 1991;151:463-468.

27. Williams ME, Connolly NK. What practicing physicians in North Carolina rate as their most challenging geriatric medicine concerns. J Am Geriatr Soc 1990;38:12.30-1234.

28. Siminoff LA. Improving communication with cancer patients. Oncology $1992 ; 10: 83-89$

29. Mizushima $Y$, Yashii T, Hoshino $\mathrm{K}$ et al. A survey regarding the disclosure of the diagnosis of cancer in Toyama Prefecture, Japan. Jpn J Med 1990;29:146-1.5.5.

30. Lavrentiadis G, Manos N, Christakis J, Semoglou C. The Greek cancer patient's knowledge and attitudes towards his diagnosis and prognosis. Psychother Psychosom 1988;49:171-178.

31. Glasser M. Alzheimer's disease and dementing disorders: Practices and experiences of rural physicians. Am J Alzheimers Disease 1993;8:28-35.

32. Rubin SM, Glasser ML, Werckle MA. The examination of physician's awareness of dementing disorders. J Am Geriatr Soc 1987;35:1051-1058.

33. Somerfeld MR, Weisman CS, Ury W et al. Physician practices in the diagnosis of dementing disorders. J Am Geriatr Soc 1991;39:172-175.

34. US Department of Health and Human Services. Alzheimer's Disease and Related Dementias. Legal Issues in Care and Treatment. A Report to Congress of the Advisory Panel on Alzheimer's Disease, 1994. 